\title{
Excretion Rate From T1 to T2 Normalized by Dose
}

National Cancer Institute

\section{Source}

National Cancer Institute. Excretion Rate From T1 to T2 Normalized by Dose. NCI

Thesaurus. Code C105451.

The excretion rate over the interval from T1 to T2 divided by the dose. 